\title{
On the Wake and Drag of Bluff Bodies*
}

\author{
ANATOL ROSHKO† \\ California Institute of Technology
}

\section{Abstract}

A modification to Kirchhoff's free streamline introduces the parameter $k=\sqrt{1-C_{p s}}$, which allows arbitrary base pressure and which must depend on the dynamics of the wake. For a cylinder of given cross-sectional shape, the drag, $C_{D}$, and the wake width, $d^{\prime}$, are functions of $k$ only. These functions are used to relate $C_{D}$ and the dimensionless shedding frequency, $S=n d / U_{\infty}$, to another number, $S^{*}=n d^{\prime} / U_{s}$, which is based on wake parameters. It is found that $S^{*} \doteq 0.16$ for all cylinders. In another approach, $k$ is evaluated by using Kármán's solution for the vortex street.

\section{SyMBols}

$c=$ distance from back of cylinder to trailing edge of interference element

$c_{D}=$ drag coefficient

$c_{p s}=$ base pressure coefficient

$d=$ cylinder diameter or breadth

$d^{\prime}=$ distance between free streamlines

$h=$ width of vortex street

$k=$ base pressure parameter $\left(U_{s} / U_{\infty}\right.$ or $\left.\sqrt{1-c_{p s}}\right)$

$l=$ longitudinal vortex spacing

$n=$ vortex shedding frequency

$R=$ Reynolds Number based on cylinder diameter $\left(U_{\infty} d / \nu\right)$

$R^{*}=$ Reynolds Number based on wake parameters $\left(U_{s} d^{\prime} / \nu\right)$

$S=$ cylinder Strouhal Number $\left(n d / U_{\infty}\right)$

$S^{*}=$ wake Strouhal Number $\left(n d^{\prime} / U_{s}\right)$

$u=$ velocity of vortices relative to free-stream velocity

$U_{\infty}=$ free-stream velocity

$U_{s}=$ velocity on free streamline at separation

$\Gamma=$ circulation per vortex

$\epsilon=$ fraction of shear layer vorticity which goes into individual vortices

$\lambda=$ wake width factor, $h / d^{\prime}$

\section{(I) INTROduction}

$\mathrm{T}$ HE PROBLEM OF THE FLOW about a bluff body is returned to, in the literature, again and again. The reason for this is clear enough - not only is the problem always of great practical importance, but it has so far not given way to theoretical treatment. In fact, there has been little new advance in the theory during the past 40 years, the contributions of Kirchhoif and Kármán still remaining the cornerstones from which almost all writers start. A large part of the theoretical work since then has been little more than

Presented at the Aerodynamics Session, Twenty-Second Annual Meeting, IAS, New York, January 25-29, 1954.

* The experiments reported were performed in the 20 - by 20 in. low-turbulence wind tunnel at GALCIT. The major portion of this work was under the financial sponsorship of the National Advisory Committee for Aeronautics. The author is indebted to Dr. H. W. Liepmann, under whose program of general turbulence research the present investigation was conducted.

$\dagger$ Research Fellow, Department of Aeronautics. elaboration and extension of those theories. But, as already pointed out by the early investigators, there seems little hope of arriving at a complete theory before something is understood of the essentially nonsteady processes in the wake immediately downstream of the cylinder-i.e., the dynamics of the vortex formation. It is unfortunate that more of the energy has not been applied in this direction. However, this part of the problem does indeed appear formidable, and one would like to progress a little further without having to tackle it directly.

Possibly not enough attention has been paid to the experimental information that has been accumulated and which is indispensable for the realistic formulation of theoretical models. The work of Fage and his associates $^{1.2}$ can be singled out as a particularly fruitful source of experimental results that have not been fully exploited.

It is the purpose of the present paper to give an overall view of the aspects of the problem which must be considered, and combined, in any complete theory. These are, principally, the potential outer flow and the wake. It is shown that a realistic representation of the potential flow, in the vicinity of the cylinder, may be obtained by a modification of Kirchhoff's method. This depends, however, on a parameter that can be determined only from a consideration of the wake. The crucial region in the "joining" of the potential part of the solution to the wake is the region of vortex formation just behind the body. Some experimental results are presented to demonstrate how critically the whole problem depends on that region. Nevertheless, even without understanding the vortex formation, the modified Kirchhoff theory may be combined with some considerations about the wake to obtain a solution. This is still semiempirical, but the empiricism is a minimum, and a useful unification of different bluff body shapes is obtained.

\section{(II) Modified Kirchhoff Method}

The main feature of flow past a bluff body is its separation from the body surface, well ahead of the rear stagnation point, and the formation of a large wake. The presence of the wake alters the flow and the pressure distribution on the body, as compared to the potential solution, resulting in a deficit of pressure on the downstream side and an excess in front. This gives a net pressure drag quite distinct from that due to friction. 
In the approach taken by Kirchhoff, the problem is to find the drag by calculating the pressure distribution. For this, one has the experimental observation that the separated boundary layer continues downstream as a free shear layer, which is well defined at first and forms a boundary of demarcation between the wake and the (distorted) potential outer flow. The problem consists then of finding the shape of this boundary (which is idealized as a surface of velocity discontinuity, or so-called free streamline) so that the potential outer flow, and thus the pressure distribution, may be calculated. The basic assumption in the Kirchhoff theory is that the pressure everywhere in the wake is the freestream pressure, $p_{\infty}$. This then is also the pressure on the "base" of the body, downstream of the separation point, as well as all along the free streamline. The magnitude of the velocity at the separation and on the free streamline must then be the free-stream value, $U_{\infty}$. Thus, in the hodograph plane, the free streamline is simply a circle and permits an elegant solution.

However, the calculated drag is considerably lower than what is observed, the discrepancy being directly due to the assumption about the base pressure, which is observed to be actually much lower than $p_{\infty}$. On the other hand, the measured pressure is remarkably uniform over the base. This, and the fact that free streamlines appear to be good representations of the free shear layers, indicates that the Kirchhoff method should be sound, provided more realistic (lower) values are chosen for the base pressure. But then the pressure in the wake can no longer be uniform, for it must eventually become $p_{\infty}$, far downstream. On the other hand, there need not be too much concern about the details of the solution far downstream, for in any case the free streamline representation will be valid for only a few diameters downstream of the body. The main aim should be to find a model that will give an accurate representation in the vicinity of the body and permit the pressure distribution on the body to be calculated.

Such a solution was first obtained by Riabouchinsky, ${ }^{3}$ who avoids the problem of the pressure distribution in the wake by constructing a closed wake, or cavity, instead of letting it extend to infinity downstream. The pressure in the cavity is uniform but may have arbitrary values. Thus a family of cavity flows is obtained in which the dimensions of the cavity, as well as the pressure distribution and drag of the body, depend on the cavity pressure as parameter. However, Riabouchinsky's solution was not exploited by him or anyone else for other than cavitation flows.

In a somewhat different approach taken by the present writer, ${ }^{4}$ the Kirchhoff method is simply modified to allow arbitrary base pressures. To demonstrate how this is accomplished, Kirchhoff's example of a flat plate set broadside to the (two-dimensional) flow is sketched in Fig. 1. The essential step, following Helmholtz and Kirchhoff, is the mapping of the flow onto the hodograph plane, from where it may be mapped onto the plane of the complex potential, and the potential flow completely determined. The vector from $o$, in the hodograph plane, to any other point represents the velocity at the corresponding point in the physical plane. Thus the velocity which at "a" has the free stream value $U_{\infty}$, decreases along $a o$ until it is zero at the stagnation point. Then it increases again, along the front of the plate, until it reaches the value $U_{s}$ at the separation point on the edge of the plate. Whereas in the Kirchhoff theory this is fixed at the freestream value, here we allow it to have an arbitrary value $U_{s}$, greater than $U_{\infty}$. Correspondingly, the pressure there, and on the base, will have the value

$$
p_{s}=p_{\infty}+(1 / 2)\left[\rho\left(U_{\infty}^{2}-U_{s}^{2}\right)\right]
$$

which is lower than $p_{\infty}$. Written as a pressure coefficient, the base pressure is

$$
C_{p s}=\frac{p_{s}-p_{\infty}}{(1 / 2) \rho U_{\infty}^{2}}=1-\left(\frac{U_{s}}{U_{\infty}}\right)^{2}=1-k^{2}
$$

where

$$
k=\left(U_{s} / U_{\infty}\right) \geq 1
$$

From $s$, in the hodograph plane, the free streamline must return to $a$, the point at infinity. Now one feels that, since the pressure is uniform over the base, it should be uniform for some distance downstream in the wake-that is, that the pressure and the magnitude of the velocity along the free streamline are constant at first. The corresponding trace in the hodograph plane is a circular arc, and we simply assume that the arc extends through a full $90^{\circ}$ as shown, until the direction is parallel to the free stream. From this point, $b$, it proceeds to a without further change of direction. In this way we obtain, in the hodograph plane, a "notched" circular hodograph that can easily be mapped into the complex potential plane, and, in the physical plane, a wake that has a definite width, $d^{\prime}$. The solution depends on the single parameter $k$-i.e., on the base pressure. The Kirchhoff solution is the limiting case, $k=1$, shown in the hodograph by the dotted circle. A typical pressure distribution for arbitrary $k$ is sketched in Fig. 1.

It is shown in Fig. 4 that if the value of $k$ (i.e., of $C_{p s}$ ) is chosen to correspond with the measured base pressure, then the calculated pressure distributions along the free streamline and on the front of the body agree well with the measured distributions, so that the measured and calculated values of drag are also in excellent agreement. On the other hand, such good agreement is not obtained when the pressure on the free streamline is assumed to start increasing immediately after separation. Therefore the assumption of a circular, notched hodograph appears to be a good one and may be applied with confidence to other cylinder shapes. In addition, it defines a definite wake width, $d^{\prime}$, which will presently prove to be most useful. The Riabouchinsky model gives equally good agreement between measured 
and calculated drag, for it also assumes constant pressure along the free streamline. It also gives a definite wake dimension for each value of $k$. Thus, for representing the early part of the flow, the two solutions are entirely equivalent, although they differ slightly in numerical detail.

The dependence of the drag $\left(C_{D}\right)$ and the wake width $\left(d^{\prime} / d\right)$ on the parameter $k$, which fixes the base pressure, is shown in Figs. 2 and 3, which have been adapted from reference 4 .

The Kirchhoff method is by no means restricted to this case of the "bluff plate." In fact, there is extensive literature on free streamline theory in which applications to many different body shapes may be found. Almost always, however, the Kirchhoff assumption is retained - that the base pressure is $p_{\infty}-$ and correspondingly the resulting values of drag are always too low. For instance, when applied to the circular cylinder, ${ }^{5,6}$ the theory gives a drag coefficient $0 . \overline{5}$, as compared to the experimental value of about 1.0.

In the application of the theory to a circular cylinder, or any smooth cylinder without edges, some additional difficulties are encountered, for in such cases the separation point is not fixed once and for all. It is assumed then that at separation the free streamline has the same curvature as the body surface. This gives a unique additional condition that makes the problem determinate. Although this does not give the "pressure bump" that is actually required to separate the boundary layer, it does give a solution that is reasonable in the large, and which may be expected to give reasonable values of the drag, provided the base pressure is chosen realistically and is not restricted to the Kirchhoff value. In reference 4 the notched hodograph has been adapted to this purpose. Just as for the bluff plate, a solution is obtained in which the drag and the wake width depend on the parameter $k$. They have been plotted in Figs. 2 and 3, along with the results for the bluff plate. These figures contain, in addition, the results for a wedge of vertex angle $90^{\circ}$.

While in each case the calculated flow and the drag correspond well with experimental results, provided $k$ is chosen appropriately, the theory now is not closed, for it does not tell how to determine the parameter $k$. This, in fact, is the position we should expect to be in, for we cannot expect to obtain a complete solution without considering the mechanics of the wake. As will be shown in the next section, the wake plays an essential part in setting the base pressure, especially that part of the wake in the first few diameters downstream of the cylinder.

\section{(III) The Coupling Region}

Although the free shear layers conform well at first with the calculated free streamlines, this agreement does not hold far downstream. Instead, the well- known periodic formation and shedding of vortices occurs, rêsulting, further downstream, in the periodic wake structure known as the Kármán vortex street. It is this transition from the potential free streamline flow to the wake regime that is at present so little understood, but which must eventually hold the key to a complete theory. The crucial nature of this region, which might appropriately be called the "coupling region" between wake and potential outer flow, may be demonstrated strikingly by some simple experiments.

In the first of these a thin partition, or splitter plate, placed along the centerline of the wake downstream of a cylinder, is found to stop the vortex shedding. The partition need not extend all the way downstream, but only for the first four or five diameters. It appears that if the two shear layers on either side of the wake cannot "see" each other in the region where they tend to roll up, then there is no stabilizing mechanism to fix a definite, periodically alternating, vortex formation, and the shear layers then break down in some other manner; independently of each other. What is more remarkable, the splitter plate has an extremely strong influence on the drag of the cylinder. In some measurements on a circular cylinder, reported in reference 7 , the drag coefficient was changed from 1.1 to 0.7 by introducing a splitter plate that extended for five diameters downstream of the cylinder. The corresponding change of base pressure coefficient, $C_{p s}$, was from -1.0 to -0.5 . In short, the prevention of vortex formation reduces the base suction.

Some indication of what is happening may be obtained from Fig. 4, which gives the corresponding pressure distributions along the wake centerline, with and without splitter plate. This not only demonstrates the change effected in the base pressure, but also shows a pressure "valley" downstream of the base, especially pronounced when the splitter plate is absent. The minimum pressure occurs about one diameter downstream of the base, just in the region where the vortices form. Actually, the pressure there fluctuates at the shedding frequency, and it is the mean value that is given in Fig. 4. But it seems clear from this that the dynamics in the region of vortex formation are such as to create a low pressure there and that the suction on the cylinder base is intimately associated with it.

This relation between vortex formation and base pressure is further illustrated in the measurements of Fig. 5, which were obtained by using a much shorter length for the splitter plate - namely, about one diameter. This is too short to completely shield the shear layers from each other, but it does interfere with the vortex formation. For instance, the shedding frequency, at fixed free-stream velocity, is changed when the position of the plate is altered along the centerline. In Fig. 5 , the shedding frequency, $n$, is given in the dimensionless form, $S=n d / U_{\infty}$, which is called the Strouhal Number. Since $U_{\infty}$ is fixed in this experi- 

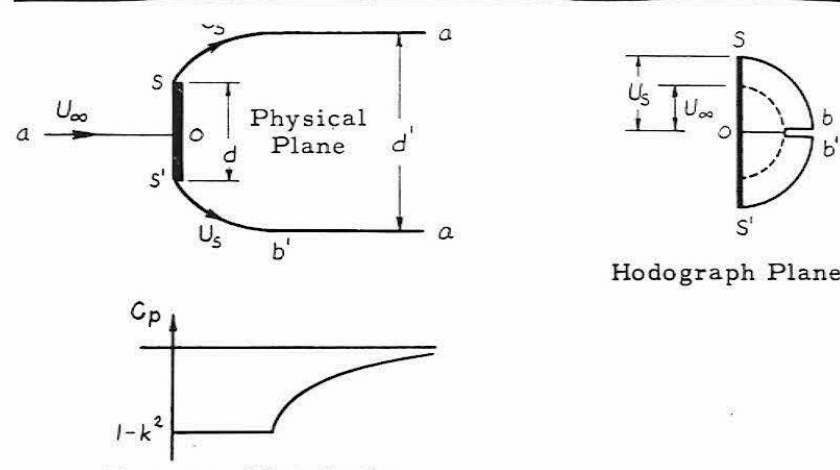

Pressure Distribution

FIG. 1.

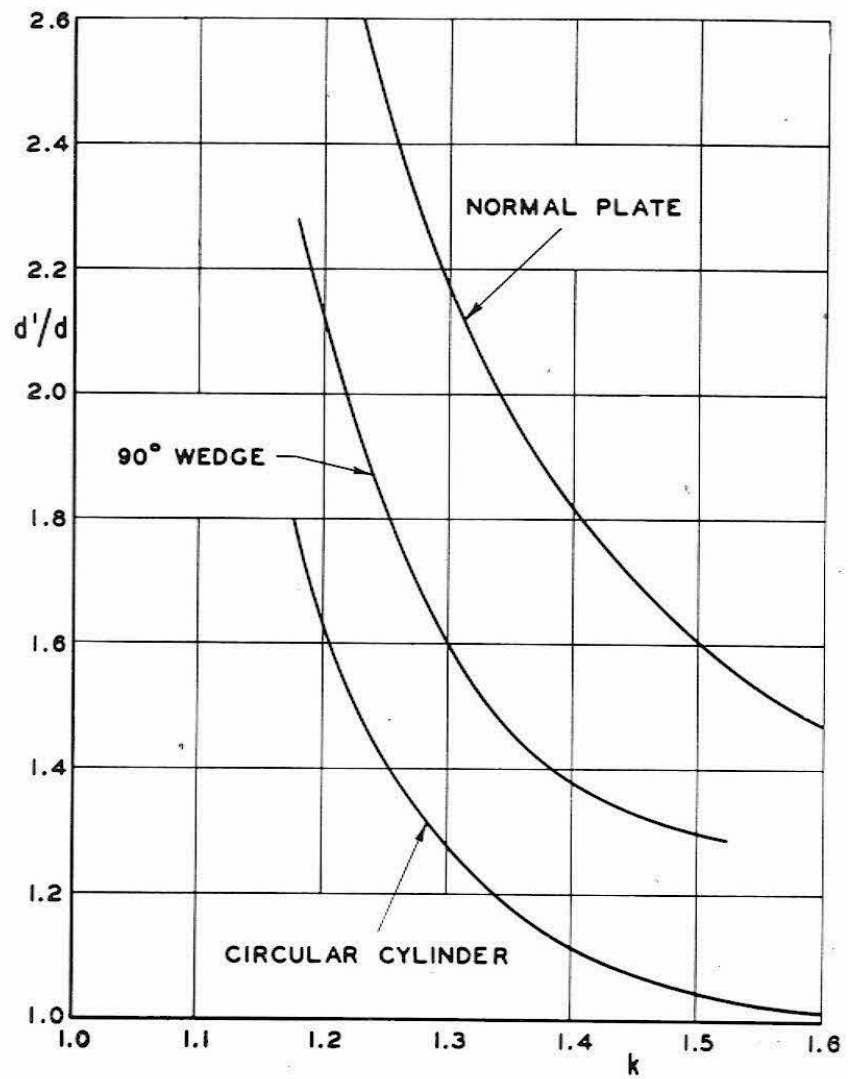

FIg. 3. Wake width.

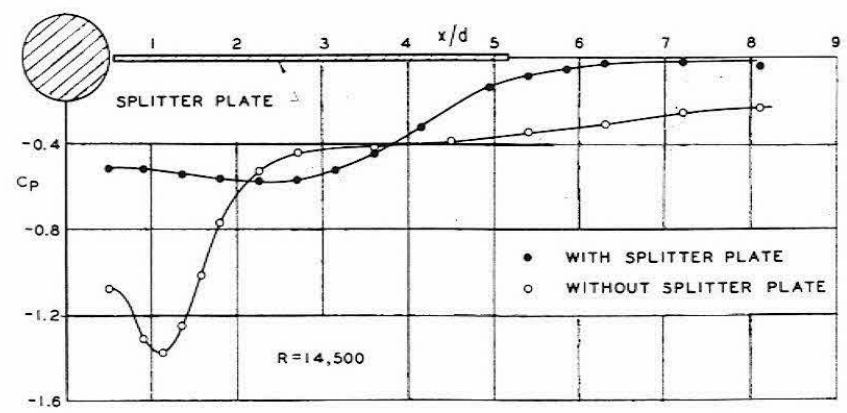

FIG. 4. Pressure on wake $£$.

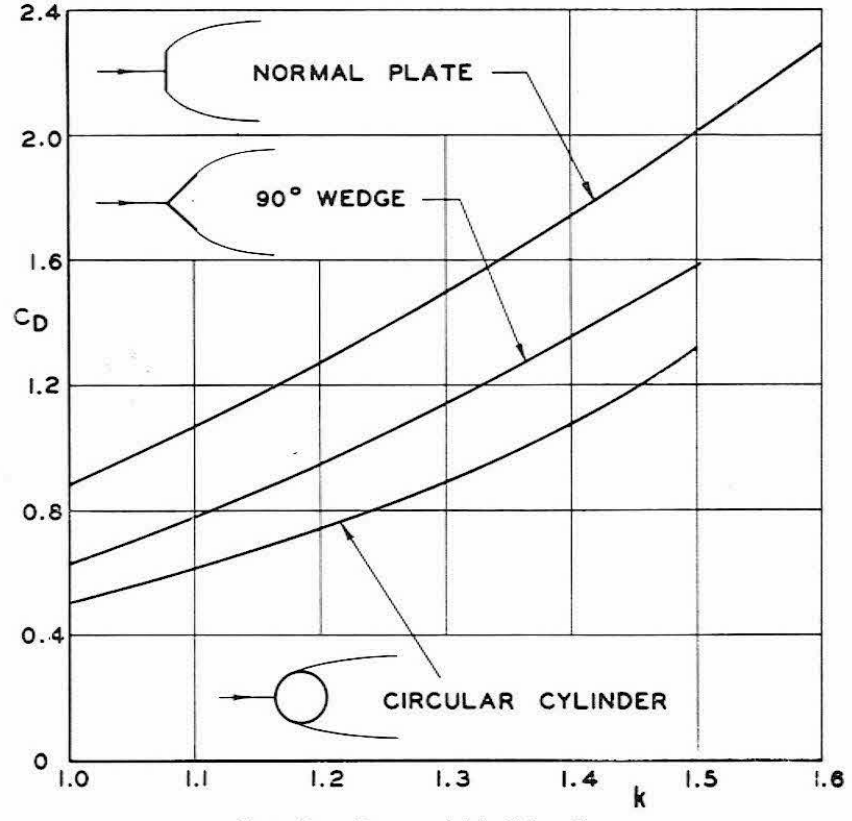

FIG. 2. Drag of bluff bodies.

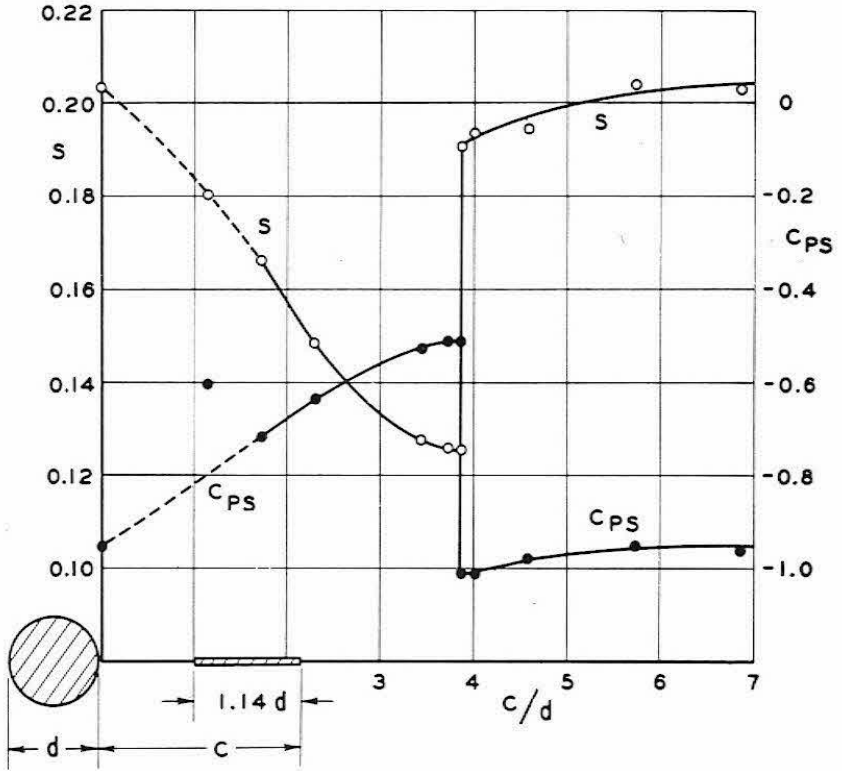

FIG. 5. Wake interference.

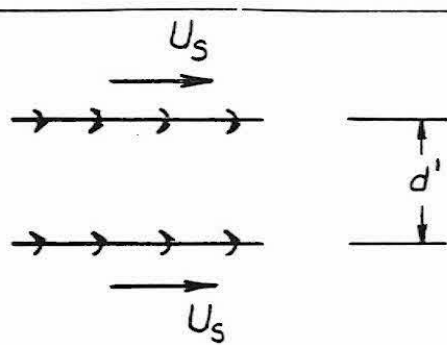

FIG. 6. 
ment, $S$ is directly proportional to the frequency. As the plate is moved downstream, the shedding frequency decreases appreciably, reaching a minimum value when the trailing edge of the plate is at four diameters downstream. Then, when the plate is moved just beyond this critical position, the shedding frequency abruptly increases, almost to its undisturbed value, corresponding to flow without interference.

The effect of the short plate in altering the shedding frequency seems to be as follows. When it is not too far from the base, the vortices form on its downstream side; this downstream shift of the formation position is accompanied by a decrease of the frequency. On the other hand, when the plate is far downstream it can have no effect, and the vortices form in their normal way. At some intermediate, critical position of the plate, the vortices have a choice of forming on either its upstream or its downstream side. Since there is no possibility for a gradual transition from one regime to the other, there must be an abrupt jump at that position.

This phenomenon is in itself interesting, but the experiment demonstrates two other important points. First, the interference is effective only in the region where the vortices form; farther downstream it has no important effect on the vortex shedding. Second, the interrelation between the vortex formation and the base pressure is clearly demonstrated by the measurements of base pressure, $C_{p s}$, which are also given in Fig. 5 and which were made simultaneously with the measurement of the shedding frequency. As the plate is moved downstream, the decrease in shedding frequency is accompanied by an increase in base pressure, which abruptly decreases again at the critical position. The increase in base pressure-i. e., decrease in suction -is no doubt partly due to the removal of the minimum pressure point from the base as the region of vortex formation is forced to move downstream. In addition, the minimum pressure itself may increase, but this point was not investigated.

The measurements given here were for the circular cylinder, but the same effects are observed for other bluff cylinders. What is clear from these experiments is that in every case the flow in the coupling region, in the first few diameters downstream of the base, is critical for the determination of the base pressure and of the overall flow. The important effect of interference elements on the vortex formation, and thus on the base pressure, indicates that there will have to be some understanding of the dynamics of these vortices. That problem is made difficult not only by its nonstationary nature, but also by the fact that, for the Reynolds Numbers of interest, the vortex formation takes place in turbulent fluid. ${ }^{8}$

This introduces the difficulties that are encountered in any turbulent flow and makes a theoretical determination of the base pressure parameter, $k$, seem rather remote at present. However, there are other possi-

\begin{tabular}{lcc}
\hline \multicolumn{3}{c}{ TABLE 1 } \\
\hline & $C_{D}$ & $S$ \\
Circular cylinder & 1.0 & 0.21 \\
$90^{\circ}$ wedge & 1.3 & 0.18 \\
Bluff plate & 1.7 & 0.14 \\
\hline
\end{tabular}

bilities for exploiting the results of the free streamline theory. Two of these are explored in the following two sections.

\section{(IV) Bluffness}

One of the features of bluff body problems which has not received sufficient attention is the idea of "bluffness" itself, though the usage of the term implies something about the associated flow characteristics. However, this usage has been mainly qualitative or intuitive. With the results of the free streamline theory it becomes possible to place these intuitive ideas on a somewhat firmer basis and to correlate them with some other experimental observations that are not so intuitive. The main features associated with bluffness may be enumerated as follows.

(1) Of two bodies having the same frontal area, the bluffer one tends to diverge the flow more, to create a larger wake, and to experience a higher drag. The results of the free streamline theory are quite in accord with this definition, as is evident from Figs. 2 and 3. In Fig. 2 the three cylinders are arranged in terms of increasing drag, in the order: circular cylinder, $90^{\circ}$ wedge, and flat plate (normal to the flow). Their bluffness is, by definition, increasing in that order. Then, in Fig. 3, the same ordering of bluffness is obtained in terms of the wake width.

(2) It is a matter of observation that bluffer cylinders have lower Strouhal Numbers. For instance, for the three cylinders that we have singled out for discussion, the typical values of Strouhal Number are given in Table 1, together with typical values of drag coefficient, for comparison. Fage and Johansen ${ }^{2}$ made the pertinent observation that the shedding frequency is related not to the cylinder dimension but to the width of the wake, being inversely proportional to it. This explains why the bluffer cylinders, which have wider wakes, have lower shedding frequencies and correspondingly lower Strouhal Numbers.

(3) In the comparisons of bluffness made in Figs. 2 and 3 and it has been implied that the value of $k$ is is the same, or nearly so, for the different cylinders. This is fairly well supported by the experimental results. For different cylinders the base pressures are approximately equal, within about 10 per cent, provided there is no interference in the wake. Whatever variations of base pressure do exist do not show any systematic variation with bluffness but appear, rather, to be related to Reynolds Number effects. Thus the variation between different cylinders is no more than what may be obtained for the same cylinder at differ- 
ent Reynolds Numbers. The remarks here apply to a range of Reynolds Numbers of about 500 to $10^{5}$ for the circular cylinder and to even higher values for the other two. This is the range in which $C_{D}$ is ordinarily described as being constant, but actually this also varies somewhat with $R$, and, in fact, the variations are associated with those in $C_{p s}$. Likewise, the Strouhal Number may be described as constant only to the same degree, its variations also being associated with the variations in $C_{p s}$. Over this Reynolds Number range the values of $C_{D}$ and $S$ are within 10 per cent of those given in Table 1 and, for our present purposes, may be assumed to be constant.

(4) The above remarks apply to cylinders with undisturbed wakes. If interference elements are placed in the wake, as described in the above experiments, then significant changes are produced in $C_{p s}$, and, correspondingly in $C_{D}$ and $S$. The important result is that, for a given cylinder, a decrease in drag is accompanied by a decrease in shedding frequency. This is at odds with the behavior indicated in Table 1 for different cylinders, but it will be seen to be entirely in agreement with the observation that shedding frequency decreases when wake width increases. For, as seen from Figs. 2 and 3, a decrease in drag of a given cylinder corresponds to an increase of wake width. This seems contrary to intuition, but it may be pointed out that the increase in wake width is accompanied by a decrease in wake velocities sufficient to give a net decrease in wake energy.

(It should be noted here that the results of Figs. 2 and 3 for the circular cylinder are not applicable when the separation point is on the back side of the cylinder, as happens at Reynolds Numbers above the critical value of about $10^{5}$. In such cases the wake width will be smaller than the cylinder dimension, and increasing wake width will correspond to increasing drag. In those cases where the separation point is on the back, the free streamline theory encounters some difficulties, which are connected with the fact that the hodograph plane becomes double sheeted.)

(5) The wake structure of different bluff bodies is similar. That is, the shape, or bluffness, of the body has no characteristic effect on the wake other than to determine its geometrical and velocity scales.

These several observations on the characteristics of bluffness may be incorporated into a simple dimensional analysis. Consider the two parallel vortex sheets sketched in Fig. 6, which are intended to be idealizations of the two free shear layers created by some bluff body. If these vortex layers roll up to form vortices, then the frequency with which this occurs should depend only on the characteristic velocity, $U_{s}$, and the dimension, $d^{\prime}$, associated with the configuration. It should not depend on the body that created it. In other words, there should be a Strouhal Number for the wake, defined as

$$
S^{*}=n d^{\prime} / U_{s}
$$

which should be universal for the vortex shedding from different bluff bodies. Of course it may be expected that there will be some variation with Reynolds Number, just as for the ordinary Strouhal Number. For the present, we propose to take this into account only roughly, by defining a wake Reynolds Number

$$
R^{*}=U_{s} d^{\prime} / \nu
$$

To take the Reynolds Number effects into account more precisely will complicate matters considerably. For instance, the shear layers set up by different bluff bodies will not be the same, even at the same $R^{*}$, for they may have had different histories up to the point where they start to roll up. Thus they may be thicker or thinner, may have become turbulent earlier or later, etc., and these features will have an important influence on the vortex dynamics. They will therefore be extremely important for the vortex dynamics and the base pressure. However, our considerations are only kinematic, and, for that, the secondary Reynolds Number effects, the details of the free streamline shape, etc., may be less important.

To confirm that such a universal wake Strouhal Number exists, some measurements were made on the three different cylinders, with and without wake interference, as follows. The wake Strouhal Number is related to the ordinary Strouhal Number by

$$
S^{*}=\frac{n d}{U_{\infty}} \frac{d^{\prime}}{d} \frac{U_{\infty}}{U_{s}}=\frac{S}{k} \frac{d^{\prime}}{d}
$$

To determine $S^{*}$, two measurements are needed. $S$ is determined in the usual way from a measurement of the shedding frequency. $\quad k$ is determined from a measurement of the base pressure, using the relation (cf. Eq. 1)

$$
k=\sqrt{1-C_{p s}}
$$

These are the only measurements needed, for the other factor, $d^{\prime} / d$, then is given by the free streamline results in Fig. 3. The relation (5) may be regarded as the essential one in the coupling between the potential flow and the wake, for it determines, for a given cylinder, the wake velocity and dimension in terms of the base pressure. The results, plotted in Fig. 7, show that there is indeed a universal relation, $S^{*}=S^{*}\left(R^{*}\right)$. Possibly the scatter is too large to show any functional trend, as has been attempted with the solid line, and one should simply assume that $S^{*}$ is constant at about the value 0.16 . The discrepancies at the lower values of $R^{*}$ are probably more than experimental and may be related to some of the secondary Reynolds Numbers referred to above. At those low values, the free shear layers may be laminar for a considerable length, before transition occurs, and this transition point may be quite different for different cylinders, even at the same $R^{*}$. 


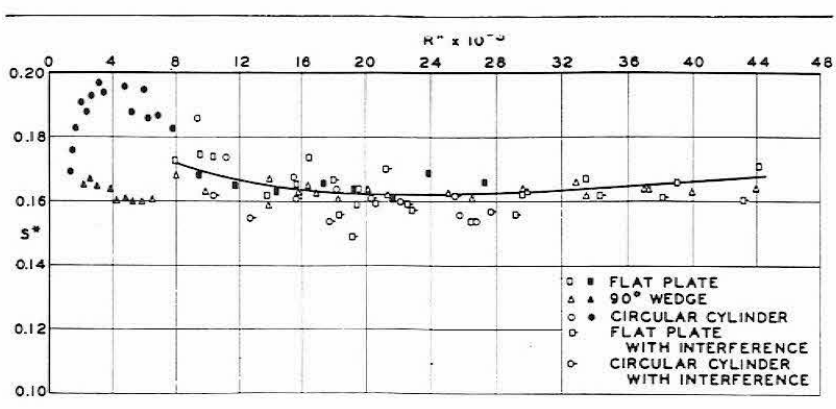

FIG. 7. Wake Strouhal Number.

It will be noted that the parameter $S^{*}$ incorporates the observation made by Fage and Johansen-namely, that the shedding frequency depends on the wake dimension rather than the cylinder dimension. $S^{*}$ is more general in that it includes also the wake velocity, $U_{s}$. This was not necessary in Fage's treatment, since all the cylinders considered had essentially the same base pressure and thus the same $U_{s}$. By including $U_{s}$, the parameter $S^{*}$ can be extended to a larger class of flows, in particular those for which the base pressure changes appreciably, as for instance with wake interference. Still another important point of difference is that the wake width is not measured, as in Fage's case, but is calculated from the free streamline theory, using the measured base pressure. This is especially convenient when the results are used in the reverse direction. For instance, it is possible to infer the drag of a given cylinder simply from a measurement of its shedding frequency, for then the factor

$$
k\left(d / d^{\prime}\right)=S / S^{*} \doteq S / 0.16
$$

is readily computed and $k$ is determined with the help of Fig. 3. The drag coefficient is then obtained from Fig. 2. (In case $S^{*}$ varies a little with $R^{*}$, the calculation may still be made easily by iteration.)

\section{(V) On Closing the Káryán Solution}

There is another way in which the potential free streamline flow may be joined to the wake. It will be recalled that in Kármán's treatment of the vortex street there are two undetermined parameters, which are usually found from experiment. These two parameters, which are essentially a dimension and a velocity, are just the ones that are needed to relate the vortex street, or wake, to whatever body produces it, as explained in the last section. Now since the free streamline theory also gives a velocity and dimension $\left(U_{s}\right.$ and $\left.d^{\prime}\right)$, which vary with $k$, it would appear to be straightforward to determine $k$ uniquely by combining the two theories. For instance, the drag must be the same, whether calculated from the free streamline theory or from Kármán's drag formula for the vortex street.

The possibility of joining the free streamline flow to the vortex street was first proposed by Heisenberg. ${ }^{9}$ He retained the Kirchhoff assumption that the base pressure is at the free-stream value, and this forced a redundancy on his model, for the drag is then fixed at the Kirchhoff value. With the free streamline theory, in which the base pressure is variable, a more realistic formulation is possible.

Unfortunately, the lack of understanding about the coupling region again precludes a strictly theoretical solution. For instance, it becomes necessary to know how much of the vorticity in the shear layers appears in individual vortices farther downstream. One might be inclined to assume that all of it does, but Fage and Johansen ${ }^{2}$ showed that such is not the case, and they estimated that only about one-half of the vorticity goes into individual vortices, the rest being canceled by mixing with opposite vorticity in the coupling region. This introduces an empirical factor into the solution, but it will nevertheless be of interest to carry it through. The details are as follows.

Along a shear layer (Fig. 6) the circulation per unit length is $U_{s}$, and the average velocity is $(1 / 2) U_{s}$, so that the rate at which circulation is transported downstream is $(1 / 2) U_{s}^{2}$. In the vortex street, where the vorticity is concentrated into individual vortices, each having circulation $\Gamma$, the rate of transport of circulation in each row is $n \Gamma$, where $n$ is the shedding frequency. But according to the observation by Fage, this is only a fraction, $\epsilon$, of the transport along the shear layer - that is

$$
n \Gamma=\epsilon U_{s}^{2} / 2=\epsilon k^{2} U_{\infty}^{2} / 2
$$

The vortices move past a given point with the velocity $U_{\infty}-u$, where $u$ is their velocity relative to the free stream, while their spacing along a row is $l$. Therefore, the frequency with which they pass the point is $\left(U_{\infty}-u\right) / l$, which must be equal to the shedding frequency, $n$. Introducing this into Eq. (7) and rearranging it in dimensionless form, gives

$$
\left[1-\left(u / U_{\infty}\right)\right]\left[\Gamma /\left(U_{\infty} l\right)\right]=\epsilon\left(k^{2} / 2\right)
$$

Then, by introducing one of Kármán's stability parameters, $\Gamma /(u l)=2 \sqrt{2}$, it is possible to eliminate $\Gamma$ from Eq. (8), and to obtain

$$
\frac{u}{U_{\infty}}=\frac{1}{2}\left(1 \pm \sqrt{1-\frac{\epsilon k^{2}}{\sqrt{2}}}\right)
$$

This may be regarded as the expression that establishes the relation between velocities in the wake with those in the free streamline flow.

Now Kármán's formula for the drag, which he obtained from a momentum calculation on the vortex street, may be written in the form

$$
C_{D}=(h / d)\left\{\left[5.65\left(u / U_{\infty}\right)\right]-\left[2.25\left(u / U_{\infty}\right)^{2}\right]\right\}
$$

Taking $h / d$ to the left-hand side and using Eq. (9) to replace $u / U_{\infty}$ results in the expression

$$
C_{D} \frac{d}{h}=1.70\left(1 \pm \sqrt{1-\frac{\epsilon k^{2}}{\sqrt{2}}}\right)+0.563 \frac{\epsilon k^{2}}{\sqrt{2}}
$$


On the other hand, the drag may also be obtained from the free streamline theory (Fig. 2) and, when combined with the wake width $d^{\prime} / d$ (Fig. 3), gives the parameter $C_{D}\left(d / d^{\prime}\right)$ as a function of $k$. We now need an expression that establishes the relation between the dimension, and for this we may simply write

$$
h=\lambda d^{\prime}
$$

where $\lambda$ is a factor that is unknown and which again expresses the lack of knowledge of the region of vortex formation. The solution is then finally determined from the equation

$$
C_{D}(d / h)=(1 / \lambda) C_{D}\left(d / d^{\prime}\right)
$$

The left-hand side is a function of $k$ and $\epsilon$ and comes from the theory of the vortex street. The right-hand side is a function of $k$ and $\lambda$ and may be calculated from the free streamline theory for each cylinder. The values of $k$ which satisfy both sides and which are the solutions are at the intersections of the two functions. In Fig. 8, the left-hand side has been plotted for several values of $\epsilon$. The right-hand side has been plotted for the three different cylinders, with $\lambda=1$. A most useful result is that all three fall on a single curve. (The branching at the higher values of $k$ may be due to the approximate methods that had to be used for calculating the wedge and the circular cylinder. An $11^{1 / 4^{\circ}}$ wedge also falls on this universal curve.)

To actually find a solution, it is necessary to know $\epsilon$, which can at present be found only empirically. But the important result is that this needs to be done for only one cylinder, since the others fall on the same curve. In other words, it tells that $k$ is the same for for all the cylinders, and this, it was noted earlier, checks well with experiment. Variations in $k$ due to variations in Reynolds Number or due to wake interference would then correspond to variations in $\epsilon$. Actually the other empirical factor, $\lambda$, in Eq. (13) should also be included in the discussion. But it will not change the fact that all the cylinders lie on the same curve; it will merely shift the position of the curve. The value $\lambda=1$, which is the only one plotted, seems to be as good as can be inferred from the experimental information for cylinders without wake interference. The vortex spacing $h$ itself is not well defined experimentally, tending to increase downstream.

Once a value of $k$ is determined, the theory will also give the shedding frequency. For instance, the universal Strouhal Number is

$$
S^{*}=\frac{n d^{\prime}}{U_{s}}=\frac{U_{\infty}-u}{l} \frac{d^{\prime}}{U_{s}}=\left(1-\frac{u}{U_{\infty}}\right)\left(\frac{d^{\prime}}{l}\right)\left(\frac{1}{k}\right)
$$

If it is again assumed that $\lambda=1$, then the factor $d^{\prime} / l$ may be replaced by Kármán's stability ratio, $h / l=$ 0.281 , so that

$$
S^{*}=(0.281 / k)\left[1-\left(u / U_{\infty}\right)\right]
$$

$u / U_{\infty}$ may be replaced from Eq. (9).

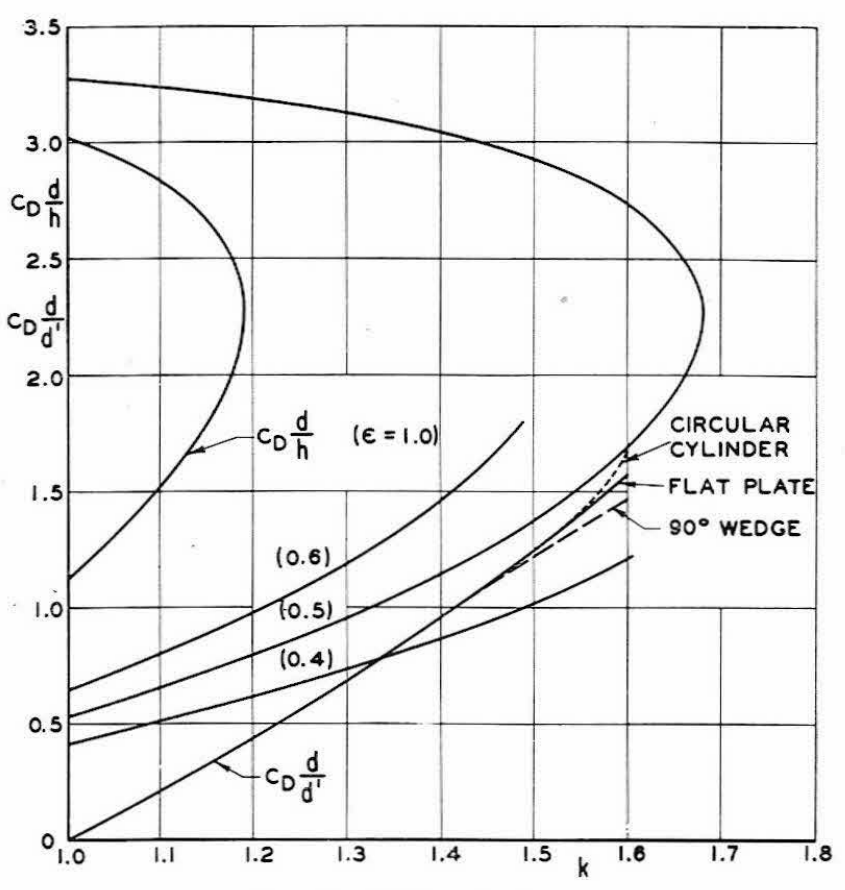

FIG, 8. Wake solutions.

For cylinders without wake interference, the base pressure coefficient for a wide range of Reynolds Numbers lies between the values -0.8 and -1.0 , tending more nearly to the latter value at high Reynolds Numbers. A suitable average value for $k$ then is 1.4. On Fig. 8 this gives an intersection at $C_{D}\left(d / d^{\prime}\right)=0.96$ and $\epsilon=0.43$. Then from Eq. (9), $u / U_{\infty}=0.18$, and, from Eq. (14), $S^{*}=0.164$, remembering that $\lambda=1$. These are all in fair agreement with the experimental information. The drag coefficients corresponding to this value of $k$ are $1.10,1.32$, and 1.74 for the circular cylinder, $90^{\circ}$ wedge, and flat plate, respectively. The corresponding Strouhal Numbers calculated from Eq. (4) are $0.206,0.167$, and 0.127 . Both drag coefficients and Strouhal Numbers compare favorably with the values listed in Table 1 .

\section{(VI) Concluding Remarks}

The experiments with the interference elements demonstrate convincingly that the critical region for the determination of the whole flow field of a bluif body is the region of vortex formation in the first four or five diameters behind the body. Some understanding of this region will be necessary for a really complete theory without empiricism.

The flow in the vicinity of the body, as well as the drag, is described adequately by the free streamline theory, provided the right value is chosen for the base pressure. Thus the theory depends on the parameter $k$, but it might be pointed out that this is the only empirical parameter. In addition, the experimental evidence indicates, and the considerations of Section V confirm, that $k$ is the same for different bluff bodies under similar wake conditions. This allows, in prin- 
ciple the solution of different bluff bodies from only a single measurement on one of them.

The free streamline theory, based on the notched hodograph or on Riabouchinsky's model, defines a wake width that varies with base pressure. This is a relationship that is extremely useful for correlating the real wake with the flow near the body, for the drag of a given body is then associated with a wake dimension and a wake velocity. It makes possible, for instance, a simple similarity treatment of the vortex shedding and the definition of a universal Strouhal Number, $S^{*}$, with which the drag may be determined from a measurement of the shedding frequency, or vice versa. Other wake phenomena might be correlated in the same way.

The results of this paper are applicable to any other two-dimensional bluff body shape if the free streamline theory is worked out for it. This sometimes presents computational difficulties, but often no more than for the cases with Kirchhoff's assumption to which a great deal of effort has been devoted. Most of the considerations of this paper could also be applied to threedimensional bluff bodies, especially bodies of revolution, if the appropriate free streamline flows could be worked out. Here the methods of conformal mappings are not available to facilitate the calculations, nor is there a theory of the wake corresponding to Kármán's theory of the vortex street.

In the experiments presented here the maximum cylinder Reynolds Numbers were about 20,000, but the same treatment should apply to much higher Reynolds Numbers. For bodies like the flat plate and wedge, in which the separation point is fixed, this should be straightforward. For cases like the circular cylinder, in which the separation point moves to the back at higher Reynolds Numbers, it is necessary to work out a suitable free streamline theory. Otherwise, the interaction between wake and cylinder should be qualitatively the same as at the lower Reynolds Numbers. For instance, some recent measurements ${ }^{10}$ on circular cylinders above the critical Reynolds Number show that the Strouhal Number is about 0.4 when the drag coefficient is 0.4 . The typical value of base pressure at these Reynolds Numbers is -0.4 , for which $k=1.2$. Assuming that the universal Strouhal Number, $S^{*}=0.16$, applies here, the wake width may be calculated from Eq. (4). It is found to be $d^{\prime} / d=$ 0.5 , which is reasonable, and supports the view that the processes in the wake are essentially unchanged at Reynolds Numbers above critical.

\section{REFERENCES}

${ }^{1}$ Fage, A., and Johansen, F. C., On the Flow of Air Behind an Inclined Flat Plate of Infinite Span, R. and M. 1104, British A.R.C., 1927; also Proc. Roy. Soc. (London), Ser. A, Vol. 116, No. 773 , pp. 170-197, September, 1927.

${ }^{2}$ Fage, A., and Johansen, F. C., The Structure of Vortex Sheets, R. and M. 1143, British A.R.C., 1927; also Phil. Mag., Ser. 7, Vol. 5, No. 28, pp. 417-441, February, 1928.

${ }^{3}$ Riabouchinsky, D., On Some Cases of Two-Dimensional Fluid Motion, Proc. London Math. Soc., Vol. 25, pp. 185-194, 1926.

${ }^{4}$ Roshko, A., A New Hodograph for Free Streamline Theory, NACA TN 3168, July, 1954.

${ }^{5}$ Brodetsky, S., Discontinuous Fluid Motion Past Circular and Elliptic Cylinders, Proc. Roy. Soc. (London), Ser. A, Vol. 102, No. 718, pp. 542-553, February, 1923.

${ }^{6}$ Schmieden, C., Die Unstetige Stromung um einen Kreiszylinder, Ingenieur-Archiv., Band 1, s. 104-109, 1929.

7 Roshko, A., On the Drag and Shedding Frequency of Bluff Cylinders, NACA TN 3169, July, 1954.

${ }^{8}$ Roshko, A., On the Development of Turbulent Wakes from Vortex Streets, NACA, TN 2913, March, 1953.

${ }^{9}$ Heisenberg, W., Die absoluten Dimensionen der Karmanschen Wirbelbewegung, Phys. Z., Vol. 23, p. 363, 1922.

${ }^{10}$ Delany, N. K., and Sorensen, N. E., Low-Speed Drag of Cylinders of Various Shapes, NACA TN 3038, November, 1953.

\section{Third U.S. National Congress of Applied Mechanics}

\section{Preliminary Announcement}

The Third U.S. National Congress of Applied Mechanics will be held at Brown University, Providence, R.I., during June $11-14,1958$. It is hoped that the scheduling of conflicting meetings can be avoided by this early announcement of the date chosen for the Congress. Further announcements concerning the preparation of papers will be made as the Congress draws nearer.

Inquiries regarding the Congress should be addressed to one of the following members of the Organizing Committee at Brown University, Providence 12, R.I.: Prof. D. C. Drucker, Secretary; Prof. E. H. Lee, Treasurer; or Prof. W. Prager, Chairman. 